\title{
Meta-analysis: re-treatment of genotype I hepatitis C nonresponders and relapsers after failing interferon and ribavirin combination therapy
}

\author{
A. G. Singal ${ }^{\star}$, A. K. Waljee ${ }^{\star}$, M. Shiffman ${ }^{\dagger}$, B. R. Bacon ${ }^{\star} \&$ P. S. Schoenfeld ${ }^{\star}, S^{\circ}$
}

*Division of Gastroenterology,

University of Michigan Medical

Center, Ann Arbor, MI, USA.

${ }^{\dagger}$ Division of Gastroenterology-

Hepatology Section, Virginia

Commonwealth University Medical

Center, Richmond, VA, USA.

"Division of Gastroenterology and

Hepatology, Saint Louis University

School of Medicine, St Louis, MO,

USA.

${ }^{\S} \vee A$ Center of Excellence for Health

Services Research, Ann Arbor, MI,

USA.

\section{Correspondence to:}

Prof. P. S. Schoenfeld, VAMC 111-D,

2215 Fuller Road, Ann Arbor, MI

48105, USA.

E-mail: pschoenf@umich.edu

\section{Publication data}

Submitted 6 May 2010

First decision 27 May 2010

Resubmitted 10 July 2010

Accepted 17 July 2010

EV Pub Online 15 August 2010

\section{SUMMARY}

\section{Background}

The efficacy of re-treating genotype I hepatitis $\mathrm{C}$ virus (HCV) patients who failed combination therapy with interferon/pegylated interferon (PEG-IFN) and ribavirin remains unclear.

\section{Aims}

To quantify sustained virological response (SVR) rates with different re-treatment regimens through meta-analysis of randomized controlled trials (RCTs).

\section{Methods}

Randomized controlled trials of genotype I HCV treatment failure patients that compared currently available re-treatment regimens were selected. Two investigators independently extracted data on patient population, methods and results. The pooled relative risk of SVR for treatment regimens was computed using a random effects model.

\section{Results}

Eighteen RCTs were included. In nonresponders to standard interferon/ribavirin, re-treatment with high-dose PEG-IFN combination therapy improved SVR compared with standard PEG-IFN combination therapy $(\mathrm{RR}=1.49 ; 95 \%$ CI: $1.09-2.04)$, but SVR rates did not exceed $18 \%$ in most studies. In relapsers to standard interferon/ribavirin, re-treatment with high-dose PEG-IFN or prolonged CIFN improved SVR $(\mathrm{RR}=1.57 ; 95 \%$ CI: 1.16-2.14) and achieved SVR rates of $43-69 \%$.

\section{Conclusions}

In genotype I HCV treatment failure patients who received combination therapy, re-treatment with high-dose PEG-IFN combination therapy is superior to re-treatment with standard combination therapy, although SVR rates are variable for nonresponders $(\leq 18 \%)$ and relapsers (43-69\%). Re-treatment may be appropriate for select patients, especially relapsers and individuals with bridging fibrosis or compensated cirrhosis.

Aliment Pharmacol Ther 2010; 32: 969-983 


\section{INTRODUCTION}

Hepatitis $\mathrm{C}$ virus (HCV) infection currently affects over 3 million people in the United States ${ }^{1,2}$ and is the leading cause of decompensated cirrhosis (e.g. ascites, hepatic encephalopathy, bleeding oesophageal varices), hepatocellular carcinoma (HCC) and liver-related mortality in the United States ${ }^{3,4}$. Furthermore, the morbidity and mortality associated with HCV infection are projected to increase steadily over the next decade. ${ }^{2}$ Although sustained viral response (SVR) rates of $54-56 \%$ have been achieved with interferon/pegylated interferon (PEG-IFN) and RBV combination therapy, ${ }^{5-8}$ individuals with genotype I HCV achieve SVR rates of approximately $40 \%$ or less ${ }^{9}$ and these patients account for approximately $75 \%$ of HCV patients in the US. Therefore, a substantial proportion of treatment-naïve HCV patients fail to achieve SVR with combination therapy and remain at high risk for decompensated cirrhosis, HCC and liver-related mortality. ${ }^{10}$

When HCV-infected patients achieve SVR before developing cirrhosis, their liver-related mortality is similar to age-matched controls. ${ }^{11}$ If HCV-infected patients with cirrhosis achieve SVR, their rates of decompensated cirrhosis [relative risk $=0.16$; 95\% CI: 0.04-0.59], HCC [relative risk $=0.21,95 \%$ CI $0.16-0.27$ ] and liver-related mortality [relative risk $=0.23$; 95\% CI: $0.10-0.52$ ] are significantly lower than similar patients who fail to achieve SVR. ${ }^{12}$ In fact, treatment failure patients with advanced fibrosis or cirrhosis develop decompensated cirrhosis at a rate of $2.9 \%$ /year, develop HCC at a rate of $3.2 \%$ /year, and suffer from liver-related mortality at a rate of approximately $2.7 \%$ /year. ${ }^{12}$ Given the morbidity and mortality associated with treatment failure, effective and safe re-treatment regimens are needed. Treatment failures include nonresponders (individuals who never clear HCV from their serum) and relapsers (individuals who temporarily clear HCV from their serum, but do not sustain viral clearance until 24 weeks after conclusion of therapy). Among nonresponders to IFN monotherapy, two meta-analyses demonstrated that re-treatment with IFN and RBV combination therapy was significantly better than re-treatment with IFN alone, but SVR was only achieved in $13-14 \%$ of patients with combination therapy. ${ }^{13,}{ }^{14}$ Among relapsers after prior IFN monotherapy, combination therapy with IFN and RBV produced SVR rates of $47-56 \%$ of patients and was superior to re-treatment with IFN alone. ${ }^{15,} 16$ However, the efficacy and safety of re-treating nonresponders or relapsers after failing combination therapy remains unclear.

Treatment of HCV-infected individuals will probably evolve to Specifically Targeted Anti-viral Therapy for hepatitis C (STAT-C) with the introduction of protease inhibitors to HCV therapy. ${ }^{17,} 18$ However, these protease inhibitors may not be widely available in the US until 2012. Therefore, selected treatment failure patients who are at risk for developing decompensated cirrhosis, HCC and liver-related mortality may be appropriate candidates for re-treatment before STAT-C therapies become available. This approach is supported by the most recent American Association for Study of Liver Disease (AASLD) and American College of Gastroenterology (ACG) treatment guidelines. ${ }^{19}$ Although multiple randomized controlled trials (RCTs) have assessed the efficacy of highdose or prolonged combination therapy vs. conventional PEG-IFN combination therapy in treatment failures, the optimal re-treatment regimen has not been established and SVR rates have not been quantified precisely.

No previous systematic review has quantitatively reviewed the study design and results of published RCTs on this topic. The objective of our meta-analysis is to assess the efficacy and safety of high-dose or prolonged PEG-IFN combination therapies for the re-treatment of HCV in patients who failed prior pegylated and nonpegylated combination therapy. These data will also provide a comprehensive assessment of SVR rates that can be attained with high-dose or prolonged combination therapy and these data can be used for comparison with SVR rates achieved with STAT-C based regimens. As genotype I accounts for the vast majority of $\mathrm{HCV}$ nonresponders and relapsers in the United States, our meta-analysis focuses on this patient population.

\section{METHODS}

\section{Literature search}

A computer-assisted search was conducted to identify potentially relevant publications in the OVID MEDLINE database on 1 September 2008. The search '1997 to September 2008' was performed using the following exploded (exp) medical subject heading (MeSH) and textwords: exp Hepatitis C/dt [Drug Therapy] AND exp retreatment $\mathrm{OR}$ recurrence $\mathrm{OR}$ treatment failuremp. [mp=title, original title, abstract, name of substance word, subject heading word] AND random allocation or random\$.mp or RCT.mp. This was then limited to humans, and a search filter designed to retrieve controlled clinical trials, systematic reviews, meta-analyses or RCTs was applied. A similar search was also performed in EMBASE from 1997 to 2008. Additional electronic searches of Digestive Diseases Week (DDW) abstracts, American Association for Study of Liver Diseases 
(AASLD) meeting abstracts, American College of Gastroenterology (ACG) meeting abstracts and European Association for the Study of the Liver (EASL) meeting abstracts from 2006 to 2008 were performed with combinations of the search terms 'hepatitis C' AND 'retreatment' or 'treatment failure' or 'recurrence' AND 'controlled trial' or 'RCT' or 'randomization'. A manual search of abstracts from these years was also performed. Manual recursive searches of references from review articles and published RCTs that met inclusion criteria were also performed. Finally, consultation with expert hepatologists was also performed to identify any additional abstracts or unpublished data.

\section{Study selection criteria}

Study inclusion criteria were (i) patient populationmajority of study patients were genotype I HCV patients who failed to achieve SVR with combination therapy (note: studies were still included if a minority of study patients were nongenotype I); (ii) intervention: comparison of two-drug regimens using currently available treatments; (iii) outcome: SVR rates; and (iv) trial design: RCT. One investigator (A.S.) reviewed the titles and abstracts of all citations identified by the literature search. Potentially relevant studies were retrieved. Two investigators (A.S., A.W.) then retrieved full texts of potentially relevant studies and applied the selection criteria. Agreement between investigators for selection of studies for the meta-analysis was $>95 \%$, and disagreements were resolved by consensus. Studies published only as abstracts were included if they had sufficient information on study design, characteristics of participants, interventions and outcomes and/or if an author of an abstract could provide this information. Data published in both abstract and full manuscript forms were derived from the full manuscript.

\section{Data extraction}

Eligible articles were reviewed in a duplicate, independent manner by two investigators (A.S., A.W.). For each study, the investigators recorded the study design, the inclusion and exclusion criteria, initial treatment regimen that study patients failed, percentage of patients with cirrhosis, percentage of patients with genotype $1 \mathrm{HCV}$, number of relapsers vs. nonresponders, re-treatment regimens including dose and duration of study medications, number of patients in each treatment arm, mean age of study patients, percentage of patients achieving the primary endpoint (SVR) and the percentage of patients who discontinued therapy because of adverse events.
Authors were contacted as necessary for any missing information.

Data regarding nonresponders and relapsers were analysed and reported separately. If studies reported SVR rates separately for genotype I patients, then these data were used for meta-analysis instead of using data for the entire patient population. Furthermore, data from trials that evaluated patients who initially failed IFN and RBV combination therapy are reported separately from trials with patients who initially failed PEG-IFN and RBV combination therapy. The primary outcome of interest, SVR, was defined as an undetectable serum HCV RNA level 24 weeks after discontinuation of therapy. Two independent reviewers (A.S. and A.W.) assessed the quality of individual RCTs based on the Jadad scoring system, ${ }^{20}$ with discrepancies resolved by a third reviewer (P.S.).

\section{Statistical analysis}

The primary aim of our study was to determine the efficacy of different re-treatment regimens for nonresponders and relapsers to prior combination therapy. The pooled response rate for an individual treatment regimen was calculated as the total number of responders divided by the total number of patients who were randomized to receive the treatment. All outcomes were analysed on an intention-to-treat basis. The pooled relative risk of SVR between different treatment regimens was computed using STATA 10 (StataCorp, College Station, TX, USA). Estimates of effect were pooled using the DerSimonian Laird method for a random effects model.

Heterogeneity was initially evaluated graphically by examination of forest plots and L'Abbe plots. We further evaluated the possibility of statistical heterogeneity by the chi-square test of heterogeneity and the inconsistency index $\left(I^{2}\right)$. A chi-square $P$-value $<0.05$ or $I^{2}$ values $>50 \%$ are consistent with the possibility of substantial heterogeneity. ${ }^{21,22}$ Sensitivity analysis, in which one study is removed at a time from the model, was performed to determine if there was possible undue influence of a single study. ${ }^{23}$ Publication bias was initially evaluated graphically by funnel plot analysis and then statistically using Begg's test. ${ }^{24}$ A symmetric funnel plot would help rule out the possibility of small studies that were not published because of unfavourable results.

\section{RESULTS}

\section{Literature search}

The MEDLINE and EMBASE search yielded 148 articles. All citations were downloaded into Reference Manager 
and then EndNote. After review of the titles and abstracts of these articles, 24 studies underwent full-text review to determine their eligibility for the meta-analysis and 14 were excluded. The remaining 10 studies (eight full text articles and two abstracts) were selected after meeting all applicable inclusion criteria (Figure 1, Table 1). Additional searches of abstracts from the annual meetings of American Association for Study of Liver Diseases (AASLD), American College of Gastroenterology (ACG), European Association for the Study of the Liver (EASL), and Digestive Disease Week (DDW) from 2006 to 2008 yielded 55 potentially relevant abstracts. After review of the titles and abstracts, 49 abstracts were excluded, and the remaining six abstracts met all inclusion criteria. Finally, recursive literature searches identified two additional full text articles that met all inclusion criteria, producing a total of 18 studies for inclusion in this meta-analysis. Two notable re-treatment trials were excluded: EPIC ${ }^{25}$ was not included as it was not a RCT, and HALT-C ${ }^{10}$ was not included because it assessed maintenance therapy with the intent of halting disease progression instead of inducing SVR. There was excellent agreement between the two reviewers $($ kappa $=1.0)$. Based on symmetrical funnel plots and statistical analysis using Begg's test $(P=0.51)$, no evidence of publication bias was ascertained.

On quality assessment, the majority of the full manuscripts $(7 / 11)$ were adequately randomized, but not double-blinded (Table S1). The other four full manuscripts were randomized, but did not describe the method of randomization in detail and received Jadad scores of 2 . The seven abstracts were possibly of similar quality, but only received Jadad scores of 1 given insufficient detail regarding randomization and withdrawals. Although none of the 18 trials was double-blinded, this finding may not be as important given the objectivity of SVR, the primary outcome of interest.

\section{Re-treatment of nonresponders to PEG-interferon and ribavirin therapy}

Two RCTs ${ }^{26,27}$ have assessed the efficacy of re-treatment in nonresponders to PEG-IFN plus RBV combination therapy (Table 1). Neither trial included relapsers. The REPEAT study ( $n=942$ patients, $91 \%$ genotype 1 ) was a four-arm RCT that evaluated the impact of high-dose induction therapy with PEG-IFN alpha 2a (360 $\mu \mathrm{g}$ /week vs. $180 \mu \mathrm{g}$ /week for 12 weeks) as well as longer treatment duration (72 weeks vs. 48 weeks). ${ }^{27}$ All four arms also received RBV $1000-1200 \mathrm{mg} /$ day. If patients did not achieve undetectable levels of HCV RNA after 24 weeks of therapy, then discontinuation of treatment was allowed. The DIRECT study ( $n=487$ patients, 95\% genotype 1) compared two different doses of Interferon alfacon-1 ('consensus interferon' or CIFN) therapy: CIFN $15 \mu \mathrm{g} /$ day plus RBV vs. CIFN $9 \mu \mathrm{g} /$ day plus $\mathrm{RBV}^{26}$ Both arms also received RBV $1000-1200 \mathrm{mg}$ /day. In the DIRECT trial, $80 \%$ of the patients were null responders to their prior course of treatment, whereas it remains

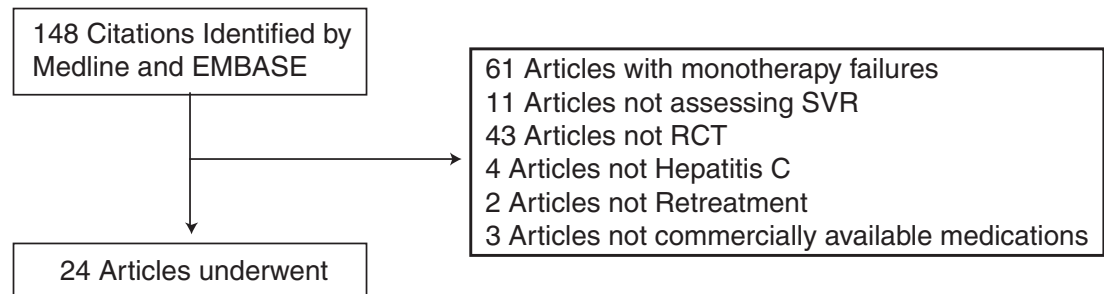

Full-Text Review

8 Full Text Articles and 2 Abstracts Included from Search 2 Additional Full Text Articles and 6 Abstracts Identified by Recursive Literature Searches 


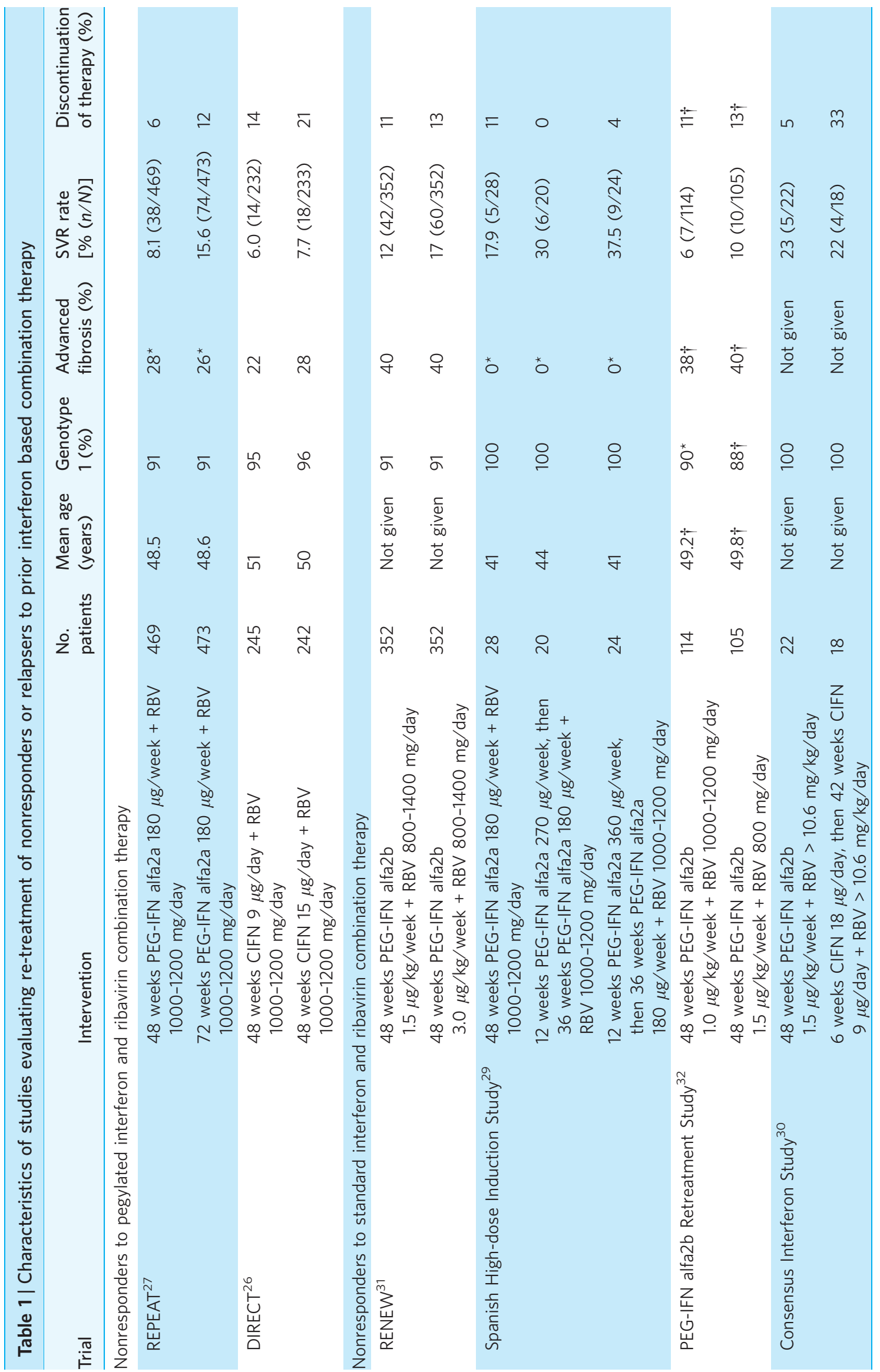


A. G. Singal et al.

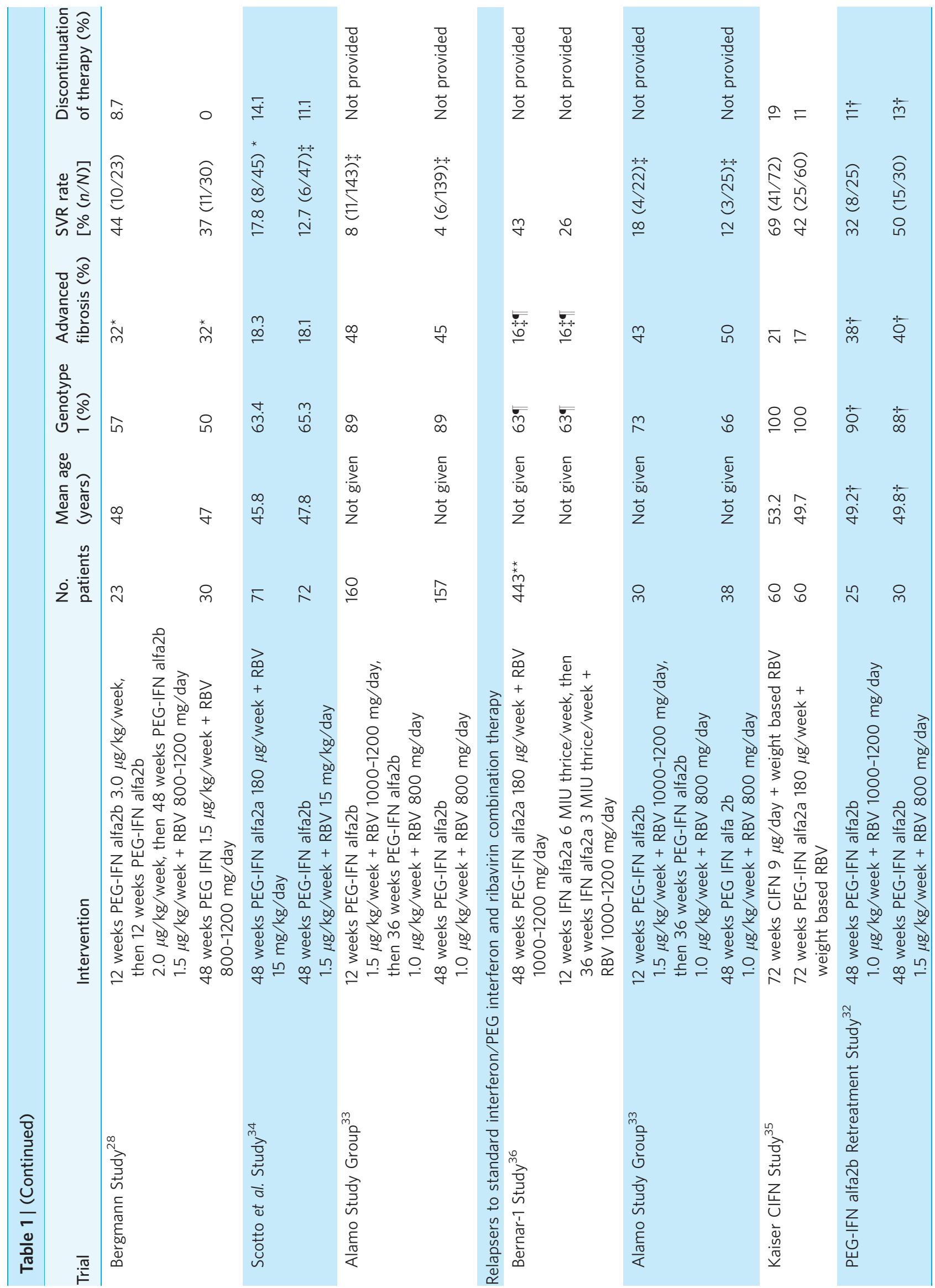




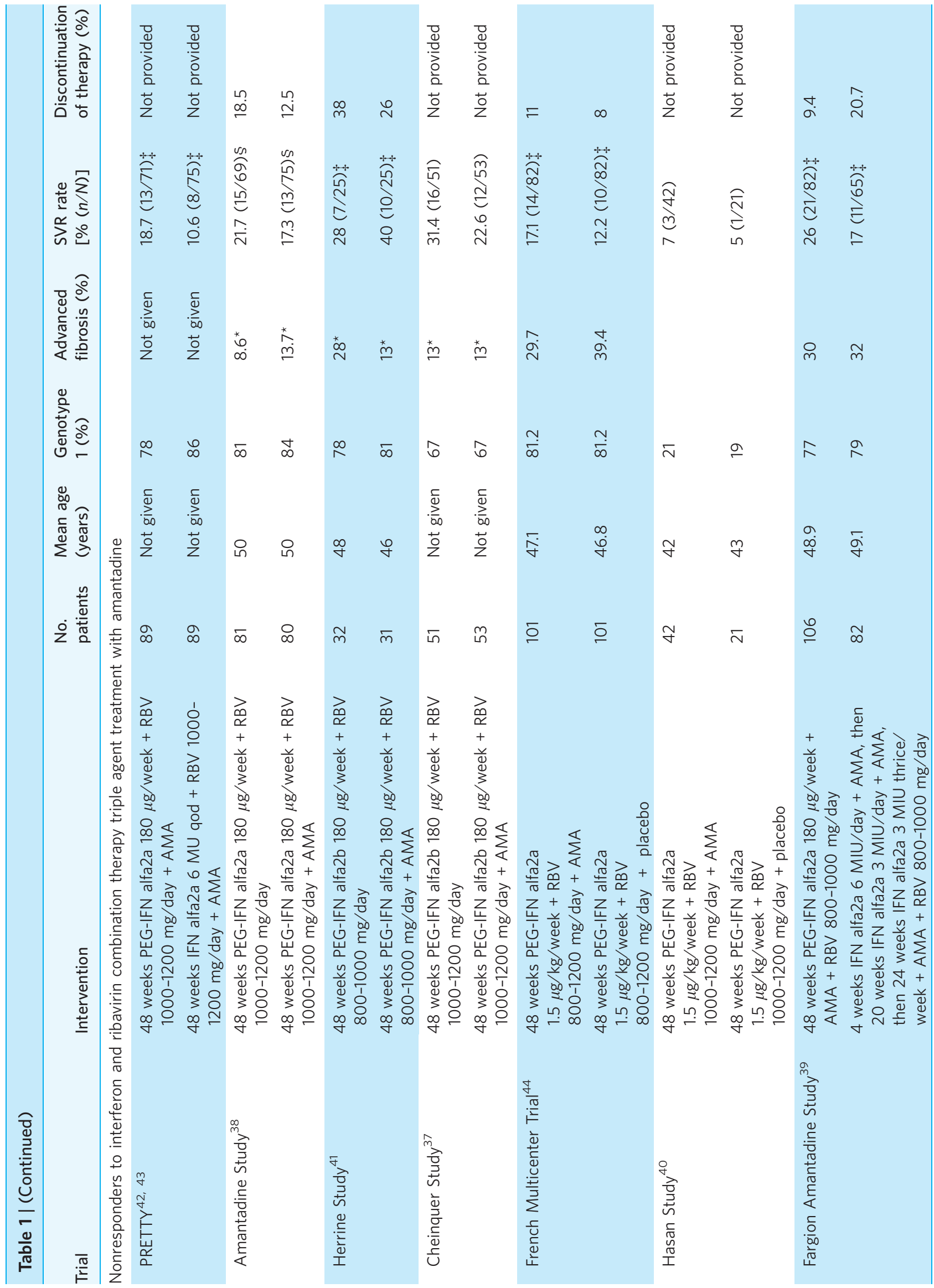



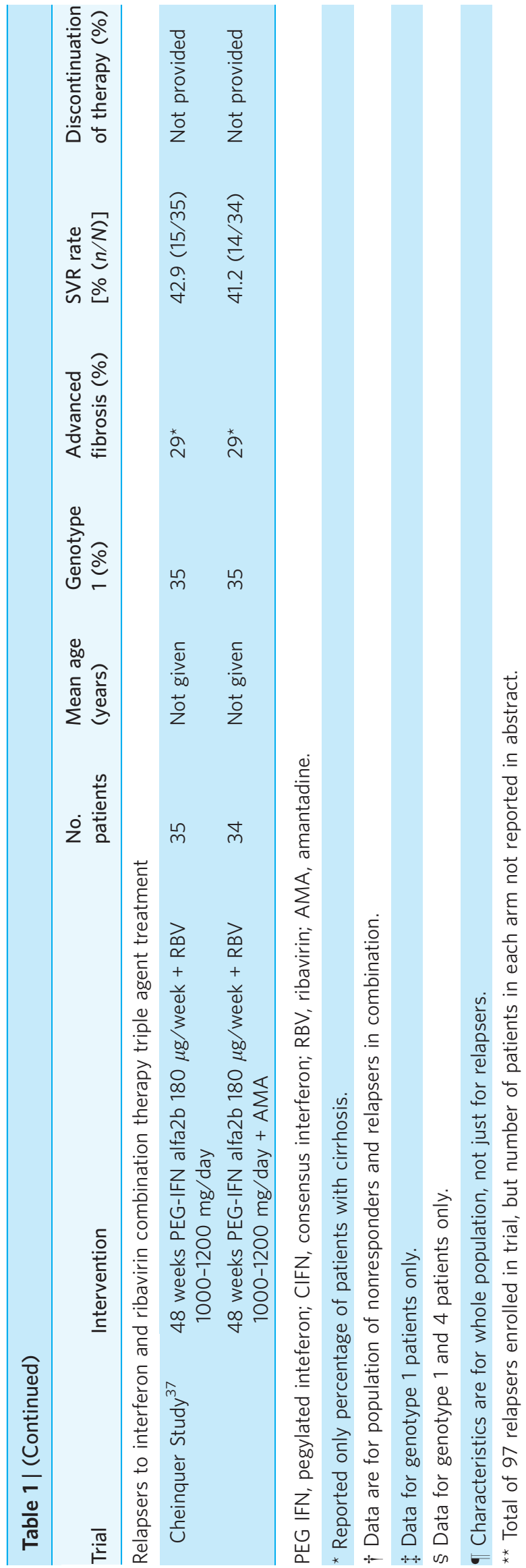

unknown how many patients were null responders vs. partial responders in the REPEAT trial.

In the REPEAT study, high-dose induction therapy had no impact on SVR rates. However, patients who were randomized to 72 weeks of PEG-IFN and RBV were more likely to achieve SVR than patients receiving 48 weeks of treatment $[16 \%(74 / 473)$ vs. $8 \%(38 / 469)$, $P<0.001 ; \mathrm{RR}=1.93 ; 95 \% \mathrm{CI}: 1.33-2.79]$. In multiple logistic regression analysis, genotype 1 patients were significantly less likely to achieve SVR than nongenotype I patients $(P<0.01)$. In the DIRECT study, patients with genotype I had SVR rates of 6.9\% (32/464) compared with $47.8 \%(11 / 23)$ for nongenotype I. Patients with genotype I randomized to CIFN $15 \mu \mathrm{g} /$ day did not demonstrate significantly higher SVR rates than patients who received CIFN $9 \mu \mathrm{g} /$ day $[7.7 \%(18 / 233) \quad$ vs. $6.0 \%$ (14/232), $\quad P=0.47 ; \quad \mathrm{RR}=1.28 ; \quad 95 \% \quad \mathrm{CI}: \quad 0.65-2.51]$. DIRECT trial data also demonstrated that 'partial' responders who achieved at least a 2-log drop during initial therapy had the 'best likelihood of responding [achieving SVR] to re-treatment with CIFN' combination therapy compared with 'null' responders. Meta-analysis of these two trials was not performed given the substantial clinical heterogeneity between the two trials.

High-dose CIFN and prolonged duration PEG-IFN arms were also more likely to lead to treatment discontinuation because of adverse events. In the REPEAT study, more patients receiving 72 weeks of PEG-IFN plus RBV discontinued therapy because of adverse events compared with patients receiving 48 weeks of therapy [12\% (55/473) vs. $6 \%(27 / 469) ; P=0.001]$. In the DIRECT study, patients treated with high dose of CIFN $15 \mu \mathrm{g} /$ day trended towards higher rates of discontinuation because of adverse events compared with patients who received CIFN $9 \mu \mathrm{g} /$ day [14.3\% (35/245) vs. $21.1 \%$ (51/242); $P=0.09$ ].

\section{Re-treatment of nonresponders to standard interferon} and ribavirin therapy

Seven RCTs have assessed the efficacy of re-treatment in nonresponders to prior standard IFN and RBV therapy. ${ }^{28-34}$ Details regarding the design of these studies are provided in Table 1 . In each of the studies, over $80 \%$ of study patients had genotype $1 \mathrm{HCV}$ with the exception of the studies by Bergmann et al. ${ }^{28}$ and Scotto et al. ${ }^{34}$ where approximately $50-65 \%$ of patients had genotype 1 disease. However, specific data on SVR rates for genotype $1 \mathrm{HCV}$ patients were not available for most studies.

Meta-analysis of these trials suggests that SVR rates for the re-treatment regimens may be dose-dependent. 
Re-treatment with higher doses of PEG-IFN is more likely to produce SVR than combination therapy regimens with conventional doses of PEG-IFN: $\mathrm{RR}=1.49$; 95\% CI: $1.09-2.04 \quad\left(Q=1.24, \quad P=0.98, \quad I^{2}=0.0 \%\right)$ (Figure 2). Assessment of individual trial results suggests that SVR rates are not significantly higher in nonresponders who failed initial therapy with standard IFN plus RBV compared with nonresponders who failed initial therapy with PEG-IFN plus RBV (Table 1). Among the four RCTs that randomized at least 100 patients, ${ }^{31-34}$ SVR rates were only $10-17 \%$ with high-dose PEG-IFN and RBV combination therapy.

Although most studies compared different dosing regimens of PEG-IFN, the Consensus Interferon study ${ }^{30}$ compared CIFN with PEG-IFN combination therapy. No difference was observed between patients randomized to CIFN vs. PEG-IFN alpha $2 \mathrm{~b}[22.2 \%(4 / 18)$ vs. $22.7 \%$ (5/22), $P=0.97$; RR $=0.98$; 95\% CI: 0.31-3.11]. Moreover, no significant difference was observed when Scotto and colleagues assessed equivalent dosing regimens of two different forms of PEG-IFN. ${ }^{34}$ In a sensitivity analysis, we removed both the Consensus Interferon study and the Scotto study from the meta-analysis, but this did not produce a significant change in the pooled relative risk of SVR (RR $=1.52$; 95\% CI: 1.09-2.14).

Discontinuation rates because of adverse events (1113\%) were similar for the treatment arms in most trials
(Table 1). However, in the Consensus Interferon study, discontinuation because of adverse events was significantly higher with CIFN than PEG-IFN [33\% (6/18) vs. $4.5 \%$ (1/22), $P=0.02]$.

\section{Re-treatment of relapsers to combination therapy}

Four RCTs ${ }^{32,33,35,36}$ studied re-treatment in relapsers after prior treatment with combination therapy (Table 1). In meta-analysis, SVR rates were increased with higher dose PEG-IFN combination therapy or prolonged (72 weeks) treatment with CIFN-based combination therapy: RR 1.57; 95\% CI: 1.16-2.14 ( $Q=1.19, P=0.76$, $I^{2}=0.0 \%$ ) (Figure 3). Several points from these studies deserve further emphasis. First, the largest $\mathrm{RCT}^{36}$ compared re-treatment with PEG-IFN combination therapy vs. standard IFN combination therapy and demonstrated that the PEG-IFN combination therapy was superior: $43 \%$ vs. $26 \%$; $P<0.001 ; \mathrm{RR}=1.93 ; 95 \% \mathrm{CI}: 1.15-3.27$ ). Second, another $\mathrm{RCT}^{32}$ showed a trend for higher SVR rates with higher dose PEG-IFN alpha $2 \mathrm{~b}$ compared with standard dose PEG-IFN alpha 2b: $50 \%(15 / 30)$ vs. $32.0 \%$ (8/25), $P=0.18$; RR $=1.56 ; 95 \%$ CI: $0.80-3.07$. Finally, the highest SVR (69\%) was achieved with a 72-week course of CIFN plus RBV. ${ }^{35}$ Discontinuation because of adverse events was not reported for three studies ${ }^{33,} 35,36$ and was only reported in combination with a nonresponder population in the fourth study. ${ }^{32}$

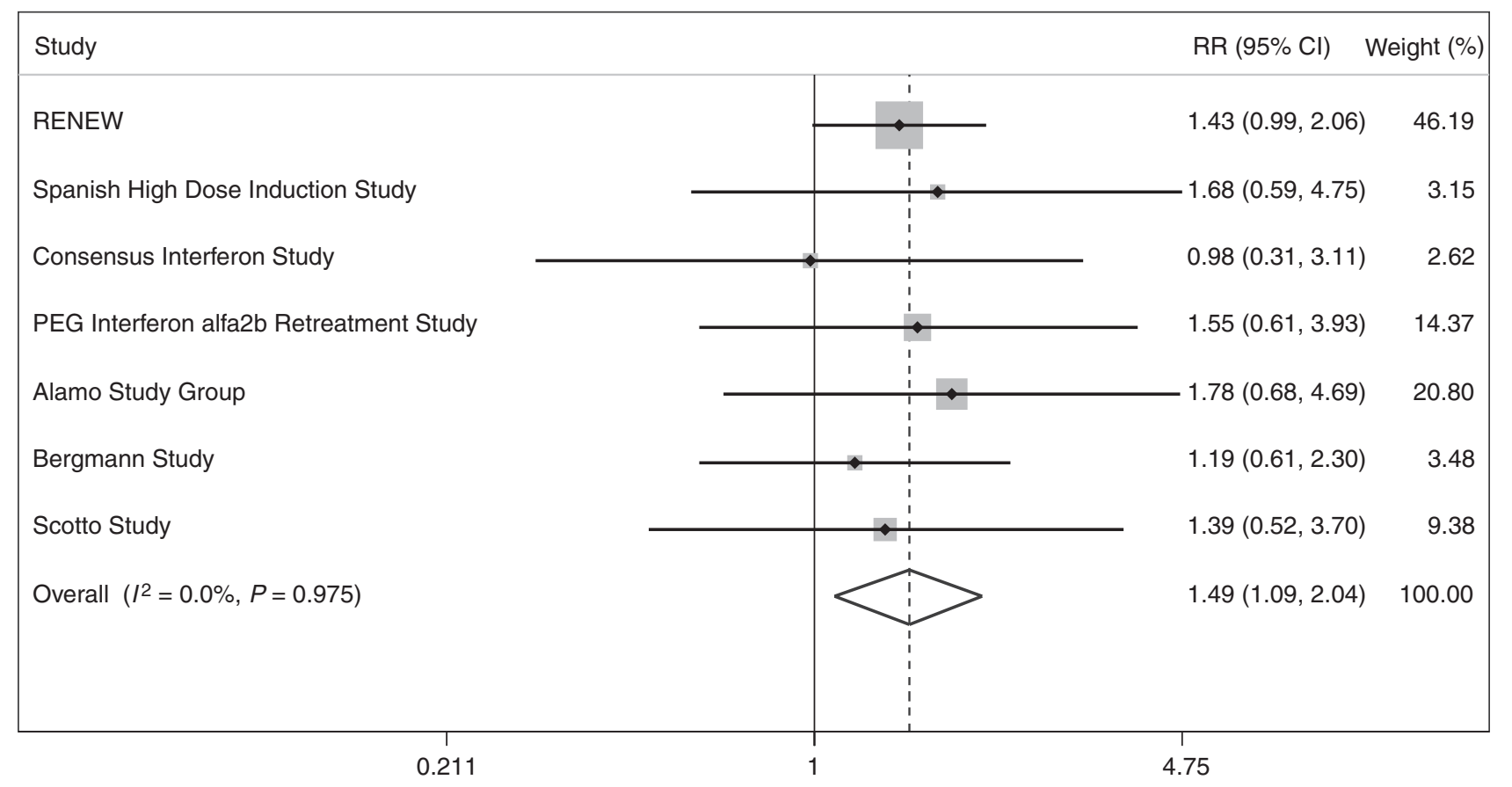

Figure $2 \mid$ Re-treatment of genotype I hepatitis C nonresponders to standard interferon and ribavirin therapy. 
A. G. Singal et al.

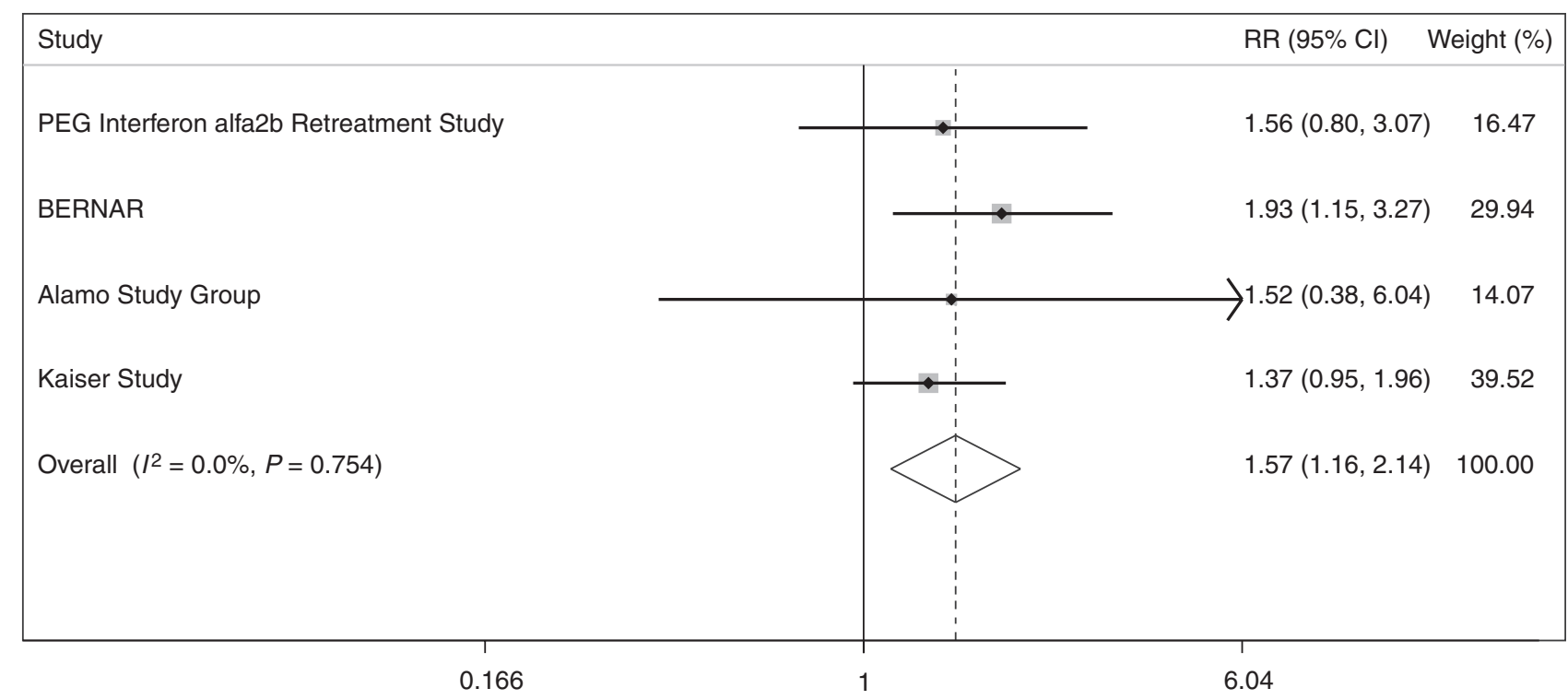

Figure 3 | Re-treatment of genotype I hepatitis C relapsers to combination therapy.

Re-treatment of nonresponders and relapsers to prior standard interferon and ribavirin therapy with amantadine

Seven RCTs $^{37-44}$ evaluated the possible benefit of adding amantadine to PEG-IFN and RBV during re-treatment (Table 1). Notably, amantadine was added to both treatment arms in two studies ${ }^{39,42,43}$ that compared different dosing regimens of PEG-IFN. With the exception of one study, ${ }^{44}$ at least $67 \%$ of the study patients had genotype I disease.

Amantadine does not appear to be beneficial based on these studies. Meta-analysis of studies that compared amantadine plus combination therapy vs. placebo (or no additional treatment) plus combination therapy $^{37}, 38,40,41,44$ demonstrated that amantadine did not improve SVR rates compared with placebo: $R R=1.07$; 95\% CI: $\quad 0.70-1.64 \quad\left(Q=3.15, \quad P=0.53, \quad I^{2}=0.0 \%\right)$. Moreover, in meta-analysis of all trials using amantadine, SVR rates were not improved for regimens that included amantadine: $\mathrm{RR}=1.24 ; \quad 95 \%$ CI: $0.90-1.72$ $\left(Q=5.58, \quad P=0.472, I^{2}=0.0 \%\right.$ ) (Figure 4$)$. Only one study provided data regarding the possible benefit of adding amantadine to PEG-IFN based combination therapy in relapser patients. ${ }^{37}$ There was no significant difference in SVR rates among relapsers with the addition of amantadine to combination therapy vs. relapsers who received more standard combination therapy: $41.2 \%(14 / 34)$ vs. $42.9 \%(15 / 35)(P=0.89)$. Discontinuation because of adverse events was not described for most of the trials, but was similar among the two treatment arms when reported. ${ }^{38,41,44}$

\section{DISCUSSION}

Most treatment-naïve genotype I $\mathrm{HCV}$ patients fail to achieve SVR with combination therapy and remain at high risk for decompensated cirrhosis, HCC and liver-related mortality. ${ }^{3,4}$ Given the morbidity and mortality associated with treatment failure, effective and safe re-treatment regimens are needed, ${ }^{12}$ but re-treatment of these patients with conventional PEG-IFN combination therapy usually has a poor outcome. Optimal re-treatment regimens for these patients, including high-dose or prolonged re-treatment regimens, have not been defined. Our meta-analysis is the first to quantify the efficacy and safety of re-treating patients who fail combination therapy with high-dose or prolonged PEG-IFN and RBV vs. conventional PEG-IFN combination therapy. It demonstrates that high-dose PEG-IFN and RBV is superior to conventional PEG-IFN combination therapy for re-treatment of nonresponders to standard interferon and RBV treatment regimens ( $R R=1.49$; 95\% CI: 1.09-2.04). Furthermore, our metaanalysis demonstrates that relapsers to initial HCV therapy can attain SVR rates of 43-69\% during re-treatment and this may be particularly useful to relapsers who already have bridging fibrosis or cirrhosis and are at risk for quickly developing sequelae of cirrhosis.

Although treatment of $\mathrm{HCV}$-infected individuals will probably advance to direct-acting antiviral (DAA) agents, 


\begin{tabular}{|c|c|c|}
\hline Study & $\mathrm{RR}(95 \% \mathrm{Cl})$ & Weight (\%) \\
\hline & & \\
\hline PRETTY & $1.72(0.76,3.89)$ & 16.10 \\
\hline Amantadine Study & $0.80(0.41,1.55)$ & 15.88 \\
\hline Fargion Amantadine Study & $1.51(0.79,2.91)$ & 20.40 \\
\hline Herrine Study & $1.43(0.65,3.15)$ & 6.95 \\
\hline Cheinquer Study & $0.72(0.38,1.37)$ & 11.47 \\
\hline French Multicenter Study & $1.34(0.63,2.87)$ & 22.27 \\
\hline Hasan Study & $\rightarrow 1.50(0.17,13.56)$ & 6.95 \\
\hline Overall $\left(I^{2}=0.0 \%, P=0.472\right)$ & $1.24(0.90,1.72)$ & 100.00 \\
\hline 0.0737 & 13.6 & \\
\hline
\end{tabular}

Figure 4 | Re-treatment of genotype I hepatitis C nonresponders and relapsers to standard interferon and ribavirin therapy with amantadine.

also known as Specifically Targeted Anti-viral Therapy for hepatitis C (STAT-C), with protease inhibitors, ${ }^{17,} 18$ these agents may not be widely available in the US until 2012 and may not be available in other countries until years later. Therefore, treatment failure patients who are at risk for developing decompensated cirrhosis, HCC and liver-related mortality may be appropriate candidates for re-treatment before DAA therapies become available, especially if they already have bridging fibrosis or compensated cirrhosis. Furthermore, the SVR data in our study will provide a comprehensive assessment of SVR rates that can be attained with high-dose or prolonged PEG-IFN combination therapy and these data can be used for comparison with SVR rates achieved with DAA based regimens.

As 2009 AASLD/ACG guidelines suggest that decisions about re-treatment should be individualized, ${ }^{19}$ clinicians need to identify treatment failure patients who are most in-need of re-treatment and those who are most likely to benefit. ${ }^{45}$ Clearly, relapsers with bridging fibrosis or compensated cirrhosis appear to be appropriate candidates for re-treatment as long as these individuals have a good understanding of the potential risks and benefits of re-treatment and express motivation to undergo prolonged or high-dose therapy. SVR rates between $43 \%$ and $69 \%$ are possible in these patients with prolonged or high-dose therapy. Nonresponders fail to achieve similar SVR rates with re-treatment, and clinicians may want to identify additional factors which are associated with successful re-treatment when making individualized decisions for patients. The EPIC trial, ${ }^{25} \mathrm{a}$ large prospective multi-centre open-label trial assessing re-treatment of 2333 patients who failed combination therapy identified other factors associated with SVR during re-treatment, including genotype $2 / 3$ disease, low baseline fibrosis score, low baseline viral load and prior standard IFN based treatment. EPIC did not assess the impact of African-American race or high body mass index (BMI), although these factors have been associated with decreased likelihood of achieving SVR in trials of treatment-naïve patients.

Other factors may also contribute to whether or not patients respond to re-treatment. Based on limited data, ${ }^{26}$ null responders (did not achieve a $2 \log 10$ drop in HCV RNA levels during the first 12 weeks of treatment and had treatment discontinued) are less likely to achieve SVR with re-treatment compared with partial responders (individuals who achieved a $2 \log 10$ drop in HCV RNA levels during the first 12 weeks of treatment). However, the studies in our meta-analysis did not consistently report adequate data regarding whether patients were partial responders or null responders to initial combination 
therapy. Future re-treatment studies should continue to report SVR rates separately for these sub-groups. Finally, HCV patients may be nonresponders because of a lack of compliance with the initial treatment regimen, inappropriate treatment holidays, or pre-mature discontinuation or inappropriate RBV dose reductions. If $\mathrm{HCV}$ RNA titers had decreased appropriately with initial therapy prior to this interruption, then these patients may be particularly likely to respond to re-treatment if they remain compliant and avoid RBV dose reductions or treatment holidays. Although it may be difficult for future trials to accurately identify patients who meet these criteria, practitioners may wish to consider re-treatment in these patients.

Based on the data in our meta-analysis, clinicians should consider the following suggestions for re-treatment regimens. For patients who are nonresponders to prior PEG-IFN and RBV combination therapy, prolonged therapy with PEG-IFN and RBV (72 weeks PEGIFN alfa2a $180 \mu \mathrm{g}$ /week + 1000-1200 mg/day RBV) results in the highest SVR rates. Similarly, high-dose PEG-IFN combination therapy regimens (48 weeks PEGIFN alfa2b $3.0 \mu \mathrm{g} / \mathrm{kg} /$ week $+1000-1200 \mathrm{mg} /$ day RBV) can be used to optimize the chance of SVR with re-treatment of nonresponders to standard IFN and RBV therapy. Re-treatment of relapsers to PEG-IFN and RBV combination therapy appears to achieve the highest SVR rates amongst the three groups, and SVR rates of $69 \%^{35}$ have been achieved with 72 weeks CIFN $9 \mu \mathrm{g} /$ day + 1000-1200 mg/day RBV (Figure 5).

When re-treating these treatment failure patients, complete viral suppression at week 12 appears to be crucial. $^{27}$ In REPEAT, patients with undetectable HCV RNA at week 12 had a $35 \%$ chance of SVR with 48 weeks of therapy and a $57 \%$ chance of SVR with 72 weeks of therapy. In contrast, those with detectable HCV RNA at week 12 had less than a $5 \%$ chance of SVR regardless of treatment duration. This suggests that complete viral suppression during the first 12 weeks of re-treatment may be critical for success. Patients with unfavourable characteristics who have a lower chance of responding to re-treatment should probably wait for DAA therapy with protease inhibitors especially if these patients only have minimal fibrosis. DAA therapy with protease inhibitors may become available in 2012, and these agents are likely to produce higher SVR rates than can be achieved with combination therapy.

Our study is strengthened by the lack of heterogeneity in our sub-group analysis. This statistical analysis provides reassurance that each individual study in sub-group

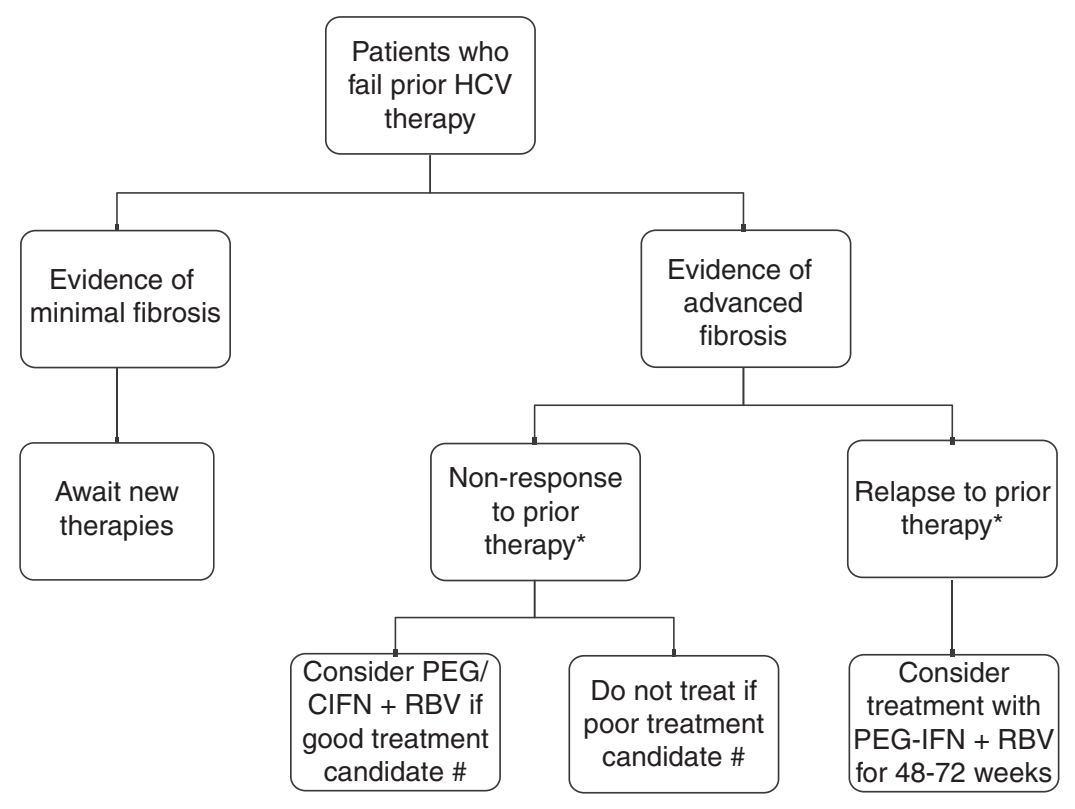

\footnotetext{
* Non- response defined as failure to clear HCV RNA from serum during therapy. Relapse defined as patients who were able to clear HCV RNA at end of therapy but did not have sustained viral response at 24 weeks after therapy was compleated.

\# Treatment candidacy determined by multiple factors such as prior viral kinetics, prior treatment asherence, and patient motivation for retreatment.
}

Figure 5 | Algorithm for re-treatment of genotype I hepatitis $C$ nonresponders and relapsers prior to hepatitis $C$ virus (HCV) therapy. 
analyses had similar study populations, treatment regimens and outcomes, making it acceptable to combine data into a single meta-analysis. However, our meta-analysis is limited by several other factors. First, seven studies in this meta-analysis are only published in abstract form. Therefore, we cannot report detailed data about frequency of adverse events, frequency of dose reductions because of adverse events, specific data on genotype I HCV patients, percentage of patients with advanced fibrosis or cirrhosis, etc. for some of the included studies. The potential impact of this on our results remains unclear. The relative lack of safety and tolerability data is particularly problematic because of these drug regimens can be associated with clinically important toxicities. Nevertheless, our metaanalysis provides a detailed summary of study design and results on this topic. Second, some RCTs published recently in abstract form ${ }^{46,47}$ did not provide adequate data to be included in this meta-analysis. Furthermore, the majority of RCTs published in abstract form did not provide sufficient data regarding SVR rates for genotype 1 patients. In these cases, data from the entire patient population were used, which could lead to an overestimation of response rates. Finally, these studies are inadequate to determine if different dosing regimens of RBV would impact SVR.

In conclusion, our meta-analysis demonstrates that high-dose PEG-IFN and RBV is superior to conventional PEG-IFN combination therapy for re-treatment of nonresponders to standard interferon and RBV treatment regimens $(\mathrm{RR}=1.49 ; 95 \% \mathrm{CI}: 1.09-2.04)$ and relapsers can attain SVR rates of 43-69\% with different regimens. If these patients have bridging fibrosis or cirrhosis and other favourable prognostic factors, then re-treatment may be appropriate while awaiting the introduction of DAA therapy.

\section{ACKNOWLEDGEMENTS}

Declaration of personal interests: All the listed authors have made substantial contributions to the conception and design of the study, acquisition of data, or analysis and interpretation of the data as well as the draft and final approval of the version to be submitted. Dr Shiffman has served on advisory boards for Anadys, Biolex, BristolMyers Squibb, Conatus, GlaxoSmith Kline, Globeimmune, Human Genome Sciences, Novartis, Roche, Romark, Schering-Plough, Valeant, Vertex and Zymogenetics. He has grant support from Biolex, Conatus, Human Genome Sciences, Idinex, Johnson/Johnson/Tibotec, Roche, Romark, Schering-Plough, Valeant, Vertex, Wyeth and Zymogenetics. He is a speaker for Roche and Schering-Plough and is a consultant for Pfizer and Roche. He is a Data Safety and Monitoring Board member for Anadys and Abbott. Dr Bacon receives research support for Schering Plough, Roche, Gilead, Bristol_Meyers Squibb, Glaxo Smith Kline, Three Rivers Pharmaceuticals, Valeant, Vertex, Human Genome Sciences, Wyeth, Echosense, Romark Labs and Intarcia. He has served on advisory boards for Schering Plough, Gilead, Glaxo Smith Kline, Three Rivers Pharmaceuticals, Vertex, Human Genome Sciences. He is a consultant for Valeant and Schering Plough, and he is a speaker for Schering Plough, Gilead, and Three Rivers Pharmaceuticals. He is a Data Safety Management Board member for Merck, Novartis, and ISIS. Dr Singal has served as a consultant for Vertex pharmaceuticals and MD-Evidence. Dr Waljee served as a consultant for Vertex pharmaceuticals and MD-Evidence. Dr Schoenfeld has worked as consultant for Vertex Pharmaceuticals and is a partner in MDEvidence. Declaration of funding interests: This research was funded by Vertex Pharmaceuticals.

\section{SUPPORTING INFORMATION}

Additional Supporting Information may be found in the online version of this article:

Table S1. Jadad quality assessment of trials.

Please note: Wiley-Blackwell are not responsible for the content or functionality of any supporting materials supplied by authors. Any queries (other than missing material) should be directed to the corresponding author for the article.

\section{REFERENCES}

1. Armstrong GL, Wasley A, Simard EP, McQuillan GM, Kuhnert WL, Alter MJ. The prevalence of hepatitis $C$ virus infection in the United States, 1999 through 2002. Ann Intern Med 2006; 144: 705-14.
2. Rustgi VK. The epidemiology of hepatitis C infection in the United States. J Gastroenterol 2007; 42: 513-21.

3. Grant WC, Jhaveri RR, McHutchison JG, Schulman KA, Kauf TL. Trends in health care resource use for hepatitis $\mathrm{C}$ virus infection in the United States. Hepatology 2005; 42: 1406-13.

4. Kim WR, Brown RS Jr, Terrault NA, El-Serag H. Burden of liver disease in the United States: summary of a workshop. Hepatology 2002; 36: 227-42. 
5. Bruno S, Stroffolini T, Colombo M, et al. Sustained virological response to interferon-alpha is associated with improved outcome in HCV-related cirrhosis: a retrospective study. Hepatology 2007; 45: 579-87.

6. Fried MW, Shiffman ML, Reddy KR, et al. Peginterferon alfa-2a plus ribavirin for chronic hepatitis $\mathrm{C}$ virus infection. $N$ Engl J Med 2002; 347: 975-82.

7. Manns MP, McHutchison JG, Gordon $\mathrm{SC}$, et al. Peginterferon alfa-2b plus ribavirin compared with interferon alfa- $2 b$ plus ribavirin for initial treatment of chronic hepatitis C: a randomised trial. Lancet 2001; 358: 958-65.

8. Simin M, Brok J, Stimac D, Gluud C, Gluud LL. Cochrane systematic review: pegylated interferon plus ribavirin vs. interferon plus ribavirin for chronic hepatitis C. Aliment Pharmacol Ther 2007; 25: 1153-62.

9. Hoofnagle JH, Seeff LB. Peginterferon and ribavirin for chronic hepatitis C. $N$ Engl J Med 2006; 355: 2444-51.

10. Di Bisceglie AM, Shiffman ML, Everson GT, et al. Prolonged therapy of advanced chronic hepatitis $\mathrm{C}$ with lowdose peginterferon. N Engl J Med 2008; 359: 2429-41.

11. Yoshida H, Arakawa Y, Sata M, et al. Interferon therapy prolonged life expectancy among chronic hepatitis C patients. Gastroenterology 2002; 123: 483-91.

12. Singal AG, Volk ML, Jensen D, Di Bisceglie AM, Schoenfeld PS. A sustained viral response is associated with reduced liver-related morbidity and mortality in patients with hepatitis $\mathrm{C}$ virus. Clin Gastroenterol Hepatol 2010; 8: 280-8.

13. Cheng SJ, Bonis PA, Lau J, Pham NQ, Wong JB. Interferon and ribavirin for patients with chronic hepatitis $\mathrm{C}$ who did not respond to previous interferon therapy: a meta-analysis of controlled and uncontrolled trials. Hepatology 2001; 33: 231-40.

14. Cummings KJ, Lee SM, West ES, et al. Interferon and ribavirin vs interferon alone in the re-treatment of chronic hepatitis $\mathrm{C}$ previously nonresponsive to interferon: a meta-analysis of randomized trials. JAMA 2001; 285: 193-9.

15. Davis GL. Combination therapy with interferon alfa and ribavirin as retreatment of interferon relapse in chronic hepatitis C. Semin Liver Dis 1999; 19(Suppl. 1): 49-55.

16. Shiffman ML, Hofmann CM, Sterling RK, Luketic VA, Contos MJ, Sanyal AJ. A randomized, controlled trial to determine whether continued ribavirin monotherapy in hepatitis $C$ virus-infected patients who responded to interferonribavirin combination therapy will enhance sustained virologic response. J Infect Dis 2001; 184: 405-9.

17. Kwo P, Lawitz EJ, McCone J. High Sustained Virologic Response (SVR) in Genotype 1 (GI) Null Responders to PEG-Interferon alfa-2B (P) plus Ribavirin $(\mathrm{R})$ when treated with Boceprevir (BOC) combination therapy. Hepatology 2009; 4: 331A.

18. McHutchison JG, Everson GT, Gordon SC, et al. Telaprevir with peginterferon and ribavirin for chronic HCV genotype 1 infection. N Engl J Med 2009; 360: 1827-38.

19. Ghany MG, Strader DB, Thomas DL, Seeff LB. Diagnosis, management, and treatment of hepatitis C: an update. Hepatology 2009; 49: 1335-74.

20. Jadad AR, Moore RA, Carroll D, et al. Assessing the quality of reports of randomized clinical trials: is blinding necessary? Control Clin Trials 1996; 17: 1-12.

21. Higgins J, Thompson SG. Quantifying heterogeneity in a meta-analysis. Stat Med 2002; 21: 1539-58.

22. Higgins JP, Thompson SG, Deeks JJ, Altman DG. Measuring inconsistency in meta-analyses. BMJ 2003; 327: 557-60.

23. Copas JB, Shi JQ. A sensitivity analysis for publication bias in systematic reviews. Stat Methods Med Res 2001; 10: 251-65.

24. Macaskill P, Walter SD, Irwig L. A comparison of methods to detect publication bias in meta-analysis. Stat Med 2001; 20 : 641-54.

25. Poynard T, Colombo M, Bruix J, et al. Peginterferon alfa-2b and ribavirin: effective in patients with hepatitis $C$ who failed interferon alfa/ribavirin therapy. Gastroenterology 2009; 136: 1618-28.

26. Bacon BR, Shiffman ML, Mendes F, et al. Retreating chronic hepatitis $\mathrm{C}$ with daily interferon alfacon-1/ribavirin after nonresponse to pegylated interferon/ribavirin: DIRECT results. Hepatology 2009; 49: 1838-46.

27. Jensen DM, Marcellin P, Freilich B, et al. Re-treatment of patients with chronic hepatitis $\mathrm{C}$ who do not respond to peginterferon-alpha2b: a randomized trial. Ann Intern Med 2009; 150: 528-40.

28. Bergmann JF, Vrolijk JM, Van der Schaar P, Vroom B, van Hoek B. Gamma-glutamyltransferase and rapid virologic response as predictors of successful treatment with experimental or standard peginterferon-alpha- $2 \mathrm{~b}$ in chronic hepatitis $\mathrm{C}$ non-responders. Liver Int 2007; 27: 1217-25.

29. Diago M, Crespo J, Olveira A, et al. Clinical trial: pharmacodynamics and pharmacokinetics of re-treatment with fixed-dose induction of peginterferon alpha-2a in hepatitis $C$ virus genotype 1 true non-responder patients. Aliment Pharmacol Ther 2007; 26: 1131-8.
30. Dollinger MM, Dridi Y, Lesske J, Behl S, Fleig WE. Efficacy of daily consensus interferon and ribavirin compared to PEG-interferon alfa-2b and ribavirin in non-responders with chronic hepatitis $\mathrm{C}$. Hepatology 2005; 42: 691A.

31. Gross J, Johnson S, Kwo P, Afdhal N, Flamm S, Therneau T. Double dose peginterferon alfa-2b with weight-based ribavirin improves response for interferon/ribavirin non-responders with hepatitis C: final results of "RENEW". Hepatology 2005; 42: 219A.

32. Jacobson IM, Gonzalez SA, Ahmed F, et al. A randomized trial of pegylated interferon alpha- $2 b$ plus ribavirin in the retreatment of chronic hepatitis C. Am J Gastroenterol 2005; 100: 2453-62.

33. Lawitz EJ, Bala NS, Becker S, Brown G, Davis M, Dhar R. Pegylated interferon alfa $2 \mathrm{~b}$ and ribavirin for hepatitis $\mathrm{C}$ patients who were nonresponders to previous therapy. Gastroenterology 2003; 124: $783 \mathrm{~A}$.

34. Scotto G, Fazio V, Fornabaio C, et al. Peg-interferon alpha-2a versus Peg-interferon alpha- $2 \mathrm{~b}$ in nonresponders with HCV active chronic hepatitis: a pilot study. J Interferon Cytokine Res 2008; 28: 623-9.

35. Kaiser S, Lutze B, Sauter B, Bissinger L, Werner C. Retreatment of HCV genotype 1 relapse patients to peginterferon/ribavirin therapy with an extended treatment regimen of 72 weeks with consensus interferon/ribavirin versus peginterferon alpha/ribavirin. Hepatology 2007; 46: 819A.

36. Nevens $F$, van Vlierberghe $H$, D'heygere F, Delwaide J, Adler M, Henrion J. Peginterferon alfa-2a $(40 \mathrm{kDa})$ plus ribavirin is as effective in patients relapsing after conventional interferon based therapy as in naive patients: results from the BERNAR-1 trial. J Hepatol 2005; 42: 214.

37. Cheinquer H, Pessoa M, Almeida P, Silva G, Patelli M. Prospective randomized study of peginterferon alpha-2a, ribavirin and amantadine vs peginterferon alpha-2a plus ribavirin, in hepatitis $\mathrm{C}$ patients now responders or relapsers to interferon-alpha and ribavirin. J Hepatol 2006; 44: S209.

38. Ciancio A, Picciotto A, Giordanino C, et al. A randomized trial of pegylatedinterferon-alpha2a plus ribavirin with or without amantadine in the re-treatment of patients with chronic hepatitis $\mathrm{C}$ not responding to standard interferon and ribavirin. Aliment Pharmacol Ther 2006; 24: 1079-86.

39. Fargion S, Borzio M, Maraschi A, Cargnel A. Triple antiviral therapy in $\mathrm{HCV}$ positive patients who failed prior combination therapy. World J Gastroenterol 2006; 12: 5293-300. 
40. Hasan F, Al-Khaldi J, Asker H, et al. Peginterferon alpha- $2 \mathrm{~b}$ plus ribavirin with or without amantadine [correction of amantidine] for the treatment of non-responders to standard interferon and ribavirin. Antivir Ther 2004; 9: 499-503.

41. Herrine SK, Brown RS Jr, Bernstein DE, Ondovik MS, Lentz E, Te H. Peginterferon alpha-2a combination therapies in chronic hepatitis $\mathrm{C}$ patients who relapsed after or had a viral breakthrough on therapy with standard interferon alpha-2b plus ribavirin: a pilot study of efficacy and safety. Dig Dis Sci 2005; 50: 719-26.

42. Mangia A, Cimino L, Persico M, Demelia L, Rumi M, Spinzi G. Enhanced response to peginteferon alfa2a-based triple therapy in previously nonresponsive chronic hepatitis $\mathrm{C}$ : final results of PRETTY study. $J$ Hepatol 2005; 42: 200-1.

43. Mangia A, Ricci GL, Persico M, et al. A randomized controlled trial of pegylated interferon alpha-2a (40 KD) or interferon alpha-2a plus ribavirin and amantadine vs interferon alpha- $2 \mathrm{a}$ and ribavirin in treatment-naive patients with chronic hepatitis C. J Viral Hepat 2005; 12: 292-9.

44. Maynard M, Pradat P, Bailly F, et al. Amantadine triple therapy for nonresponder hepatitis $\mathrm{C}$ patients. Clues for controversies (ANRS HC 03 BITRI). J Hepatol 2006; 44: 484-90.

45. Jensen D, Marcellin P. Reply to Treatment of Nonresponder Patients with
Chronic Hepatitis C: To Repeat or Not to Repeat? That is the Question. Gastroenterology 2009; 137: 1524.

46. Hezode C, Foucher J, Bronowicki J, Leroy V, Tran A. Efficacy and Safety of an intensified regimen of pegylated interferon alfa-2a plus ribavirin in $\mathrm{HCV}$ genotype 1-infected patients who did not respond to a prior standard regimen of pegylated interferon alfa-2a plus ribavirin: an interim analysis of the multicentric, randomized, controlled SYREN trial. Hepatology 2008; 48: 1148A.

47. Kaiser S, Lutze B, Hass HG. Treatment of peginterferon/ribavirin nonresponders with daily dosingof consensus interferon and ribavirin: results from the german consensus interferon multicenter study. Hepatology 2008; 48: 1143A. 